\begin{tabular}{|c|c|c|}
\hline & Int.J.Curr.Microbiol.App.Sci (2016) 5(8): 394-401 & \\
\hline & International Journal of Current Microbiology and Applied Sciences & \\
\hline & ISSN: 2319-7706 Volume 5 Number 8 (2016) pp. 394-401 & \\
\hline $\begin{array}{l}\text { EXCELLENT } \\
\text { PUBLISHERS }\end{array}$ & & www ijemas.com \\
\hline
\end{tabular}

Original Research Article

http://dx.doi.org/10.20546/ijcmas.2016.508.042

\title{
Antimycobacterial Activity of Norbornene-Polyethylene Glycol, Isoniazid and Rifampicin Nanocarrier towards Mycobacterium tuberculosis
}

\author{
K. Kalaiselvi ${ }^{1}$, V. Mangayarkarasi ${ }^{1}{ }^{*}$, N.S. Gomathi ${ }^{2}$, \\ R.M. Shivshankar ${ }^{3}$ and Raja Shunmugam ${ }^{3}$ \\ ${ }^{1}$ Department of Microbiology, SRM Medical College Hospital and Research Centre \\ (SRM MCH\&RC), Kancheepuram, Tamil Nadu, India \\ ${ }^{2}$ Department of Bacteriology, National Institute for Research in Tuberculosis (NIRT), \\ Chetpet, Tamil Nadu, India \\ ${ }^{3}$ Polymer Research Centre, Department of Chemical Science, Indian Institute of Science \\ Education and Research Kolkata (IISER K), Mohanpur 741246, India \\ *Corresponding author
}

Keywords

Norbornene,

Polyethylene

Glycol, Covalent

binding,

Nanocarrier,

Isoniazid,

Rifampicin.

Article Info

Accepted:

17 July 2016

Available Online:

10 August 2016

\section{A B S T R A C T}

The digital world that we live today is filled with innovations and advanced technologies, but still the role of medical sectors in preventing infectious diseases like Tuberculosis is feeble- minded. One of the advanced technologies so called Nanotechnology plays a vital role in research for the diagnosis and treatment of Tuberculosis, thus preventing side effects and drug resistance. The present study aimed at evaluating, Norbornene derived Isoniazid Co polymer (NOR-PEG-INH) and Norbornene derived Rifampicin Co polymer (NOR-PEG-RIF), a novel nanocarrier along with the drug for Tuberculosis with targeted drug delivery and longer bioavailability. The nanocarrier along with the drug preparation and their property were studied by the collaborated institute, Indian Institute of Science Education and Research (IISER) by covalent binding method. The invitro activity of the drug was evaluated using absolute concentration method to observe the Mininium Inhibitory Concentration (MIC) of the drug against H37Rv control strain of Mycobacterium tuberculosis (MTB) and clinical isolates of Mycobacterium tuberculosis from patient samples. The results showed that NOR-PEG-INH and NOR-PEG-RIF, the nanocarriers of Isoniazid and Rifampicin were able to show the lower MIC to inhibit Mycobacterium tuberculosis.

\section{Introduction}

India is the country with highest burden of Tuberculosis (TB) patients with 2. 2 million cases annually (WHO, 2015). The current tuberculosis treatment regimens under Directly observed therapy short course (DOTS) although highly effective, are far from ideal (Alliance, 2015).
Using the optimal combination of available four drugs (Isoniazid, Rifampicin, Pyrazinamide and Ethambutol) the duration of treatment required for curing patients cannot be reduced below 6 months (Richard et al., 2005). Long duration and complexity of the treatment results in non-adherence to 
the treatment which leads to critical response like failure or relapse or Multidrug resistance Tuberculosis (MDR-TB) in patients (Jing et al., 2008).

Since 1960 new drugs for Tuberculosis are being developed but still we could not find a better alternative for the effective four drugs (Isoniazid, Rifampicin, Pyrazinamide and Etambutol). Isoniazid and Rifampicin plays an important role in inhibition and killing of Mycobacterium tuberculosis this indicates that lot of research is needed in this arena for a better alternative or modification of these two drugs. The development of newer TB treatment regimens have several limitations like the new compounds when they get to the animal testing stage the number of laboratories capable of in vivo testing is sadly minimal (Ormer, 2011). The new compound should show activity against drug sensitive and drug resistant mycobacterium, active against non replicating mycobacterium (Latent Tuberculosis) and absence of cross resistance with existing therapeutic agents for TB (Ming et al., 2014).

Food and drug administration(FDA) and European Medicine Agency(EMA) has recently approved two anti- TB drugs namely Bedaquiline and Delamanid but the duration of treatment and side effects of drugs has to be studied further (Skripconda et al., 2013; Diacon et al., 2014). Currently there is an urgent need to improve treatment by either enhancing the application of existing agents with nanocarriers or introducing new drugs. The evolution of drug delivery systems has produced novel solutions to reduce the side effects and duration of treatment using these four drugs through nanotechnology (Svetlana, 2005).

Nanotechnology works on matter at dimensions in the nanometer scale length (1$100 \mathrm{~nm}$ ) and thus can be used for a broad range of applications and the creation of various types of nano materials and nano devices (Pratima, 2015). Nanotechnology has provided a huge improvement to pharmacology through the designing of drug delivery systems able to target phagocyte cells infected by intracellular pathogens, such as Mycobacteria, for an example invitro study was done on Tyloxapol niosomes for encapsulation, stabilization and dissolution of anti-tubercular drugs formulation and the study showed that reduce dose-related drug toxicity but bioavaibility of the drugs was shorter (Mehta and Neha, 2013).

Norbornene derived doxorubicin polymeric micelle with hydrazone linker shows excellent performance inside the cell as an efficient drug delivery system and longer bioavailability (Shivashankar et al., 2014a). Amphilic Norbornene derived thiobarbiturate homopolymeric vesicles have great scope in the field of medicine as they symbolize themselves as promising carriers for the stimuli triggered intracellular delivery of hydrophobic drugs (Shivasankar et al., 2014a; Shivasankar et al., 2014b).

Narborornene derived antituberculosis drugs demonstrated that this can be a multi frontline TB drugs for potential therapeutic treatment (Shivasankar et al., 2011). It has stability under physiological condition and depending on acidic $\mathrm{pH}$ condition the drug releases and has the feasibility of potential drug delivery in macrophage compartments but the interaction of Norbornene along with isoniazid and rifampicin towards Mycobacterium tuberculosis has to be studied detailed (Vijayakameswara, et al., 2012).

Thus the aim of the study was to demonstrate Invitro antimycobacterial activity of Norbornene-Polyethylene glycol Isoniazid and Rifampicin nanoparticles towards Mycobacterium tuberculosis. 


\section{Materials and Methods}

The plain nanocarrires and the Isoniazid and Rifampicin drugs with nanocarriers were received from Department of polymer chemistry, IISER, Kolkata, India. Isoniazid and Rifampicin separately were attached to the Norbornene monomers through a stimuli responsive linker by Covalent binding method. Figure 1 and 2 shows the binding of anti-TB drugs with Norbornene.

\section{Antimycobacterial susceptibility testing- Inoculum preparation}

H37Rv strain of $M$. tuberculosis received from NIRT (National institute for Research in Tuberculosis) was used as the standard strain and 60 isolates from the patients who were clinically diagnosed with pulmonary tuberculosis were included in this study. The Institute ethical committee approval and consent from the pulmonary tuberculosis patients were obtained. Two sputum samples, spot sample and early morning samples were collected in a universal container to identify and isolate the organism.

The tubercle bacilli were identified in sputum samples by Ziehl-Neelsen staining and cultured on Lowenstein Jensens Medium (LJ) after purification with Modified Petroffs Method using $4 \% \mathrm{NaoH}$. H37Rv, the control strain was grown within 3 weeks in LJ; other clinical isolates were grown in different period from 2 weeks to 8 weeks. All the Mycobacterium tuberculosis isolates were confirmed by Niacin, Catalase and Nitrate test with control medium of Para nitro benzoic acid (PNB) Medium

\section{Drug preparation}

As per the ICMR Manual $20 \mathrm{mg}$ of NORPEG-INH and NOR-PEG-RIF was dissolved in $20 \mathrm{ml}$ of sterile distilled water and $20 \mathrm{mg}$ of Dimethyl formamide respectively to make up $10,000 \mu \mathrm{g} / \mathrm{ml}$ which serves as a stock solution, from the stock solution working solution was prepared by further dilution. The stock solution was maintained at $-20^{\circ} \mathrm{C}$, working solution was used for the incorporation of the drug into Lowenstein Jensen's Medium.

\section{Absolute concentration method}

To calculate efficacy of the drugs, drug susceptibility test was performed using absolute concentration method. The following concentration of anti-TB nano drugs was used in this study; 0.025, 0.05, $0.1,0.2,0.5,1,2.5,5 \mu \mathrm{g} / \mathrm{ml}$ for INH $1,2,4,8$, $16,32,64,128 \mu \mathrm{g} / \mathrm{ml}$ for RIF. Drug free media and media containing graded concentration of the drugs were inoculated with the standard strain H37Rv of MTB and clinical isolates of Mycobacterium tuberculosis. Readings were taken on 28th day (NIRT manual, 2010).

\section{Control study}

Antimycobacterial activity of Plain Norbornene, dimethyl formamide, polyethylene glycol were separately tested as a control with different concentration from 0.01 to $100 \mu \mathrm{g} / \mathrm{ml}$ using H37Rv strain of MTB to show the nanocarrier has any antimycobacterial activity.

\section{Statistical analysis}

The MIC results were presented as mean value. Parametric $t$ test was used to employed to calculate the significance of difference between Plain Isoniazid: NORPEG-INH and Plain Rifampicin: NOR-PEGRIF for clinical isolates of MTB and $\mathrm{H} 37 \mathrm{Rv}$ strain of MTB. Data were analyzed at $95 \%$ confidence interval. 


\section{Results and Discussion}

Minimum Inhibitory concentration tested from $0.01-100 \mu \mathrm{g} / \mathrm{ml}$. The nanocarrier showed different MIC on the control H327Rv strain explianed in Table 1.

Table 2 explains about the drug susceptibility testing of newer drug and plain drugs towards $\mathrm{H} 37 \mathrm{Rv}$ strain of MTB Test were done repeatdly for two times to get better results. the MIC of Norbornene based INH was around $1 \mu \mathrm{g} / \mathrm{ml}$ and for plain INH was around $0.025 \mu \mathrm{g} / \mathrm{ml}$. the MIC of Norbornene based RIF was around $4 \mu \mathrm{g} / \mathrm{ml}$ and for plain drug RIF was around $32 \mu \mathrm{g} / \mathrm{ml}$.

Table 3 shows the Minimum inhibitory concentration (MIC) of clinical isolates with plain TB drugs and Norbornene based TB drugs. It was observed that NOR-PEG-RIF showed anti-TB activity with lower concentration . By using correleration study we found that there is no significant antimycobacterial activity of NOR-PEGINH $(\mathrm{P}=.14)$ but there is a significant antimycobacterial activity of NOR-PEG-RIF $(\mathrm{P}=.00)$ in comprison with Rifampicin.

The result contains the Minimum Inhibitory Concentration obtained by absolute concentration method of antimycobacterial susceptibility testing of control strain of $\mathrm{MTB}, \mathrm{H} 37 \mathrm{Rv}$ and 60 clinical isolates of MTB with plain nanocarriers, plain INH and
RIF and co polymer of nanocarrier with Isoniazid and Rifampicin.

Norbornene based nanaocarriers have a capacity to incorporate with antituberculous drugs and form a copolymer. So it is possible to demonstrate the antimycobacterial susceptibility activity invitro. The presence of antimycobacterial activity of INH-NPS against Mycobacterium tuberculosis indicates that the formed copolymer does not affect the original mechanism of anti -TB activity of the drugs Isoniazid and Rifampicin activity. The copolymer and the anti TB directly interacts with Mycobacterium tuberculosis the results were similar to that of another study done using analogue of Isoniazid (Svetlana, 2005).

Isoniazid and rifampicin are the main drugs involved in MDR-TB, the nanocarrier of these drugs will inhibit the growth of the organism and thereby lower the MIC to use effectively as Anti-TB drugs. Also Norbornene nanocarrier with Rifampicin was found to be 3 times more active than plain Rifampicin.

If the minimum inhibitory concentration of the nanocarrier based drug is lesser than the cut off value of $64 \mu \mathrm{g} / \mathrm{ml}$ for Rifampicin, 0.2 $\mu \mathrm{g} / \mathrm{ml}$ for isoniazid, then we can interpret that the organism is susceptible to the drug (NIRT Manual, 2010).

Table.1 Antimycobacterial testing of nanocarriers with H37Rv control strain of MTB

\begin{tabular}{|l|l|l|}
\hline Componenents & Strain & MIC in $(\boldsymbol{\mu g} / \mathbf{m l})$ \\
\hline Norbornene & H37Rv & 25 \\
\hline Polyethylene glycol & H37Rv & 100 \\
\hline Dimethyl formamaide & H37Rv & 50 \\
\hline
\end{tabular}


Table.2 Drug susceptibility testing of nanocarrier with drug and plain drugs towards H37Rv strain of MTB

\begin{tabular}{|l|l|l|l|l|}
\hline $\begin{array}{l}\text { Antimycobacterial } \\
\text { drugs }\end{array}$ & $\begin{array}{l}\text { Isoniazid } \\
\text { Plain drug I }\end{array}$ & NOR-PEG-INH & $\begin{array}{l}\text { Rifampicin } \\
\text { Plain drug II }\end{array}$ & NOR-PEG-RIF \\
\hline $\begin{array}{l}\text { Concentration of } \\
\text { drugs used in }\end{array}$ & $0.025,0.05$, & $0.025,0.05$, & $1,2,4,8,16,32,64,128$ & $1,2,4,8,16,32,64,128$ \\
$\boldsymbol{\mu g} / \mathbf{m l}$ & $0.1,0$. & $0.1,0$. & & \\
\hline $\begin{array}{l}\text { MIC of H37Rv } \\
\text { strain }(\boldsymbol{\mu g} / \mathbf{m l})\end{array}$ & $2,0.5,1,2.5,5$ & $2,0.5,1,2.5,5$ & & $\mathbf{4}$ \\
\hline
\end{tabular}

NOR- Norbornene, PEG- Polyethylene glycol, INH- Isoniazid, RIF- Rifampicin

Test were done repeatdly for two times to get better results. the MIC of Norbornene based INH was around $1 \mu \mathrm{g} / \mathrm{ml}$ and for plain INH was around $0.025 \mu \mathrm{g} / \mathrm{ml}$. the MIC of Norbornene based RIF was around $4 \mu \mathrm{g} / \mathrm{ml}$ and for plain drug RIF was around $32 \mu \mathrm{g} / \mathrm{ml}$.

Table.3 Standardisation / Minimum inhibitory concentration (MIC) of clinical isolates with plain TB drugs and Norbornene based TB drugs $(n=60)$

\begin{tabular}{|c|c|c|c|c|c|c|c|c|c|c|c|c|c|c|c|c|}
\hline \multirow[t]{2}{*}{ Drugs } & \multicolumn{8}{|c|}{ Isoniazid Concentration in $\mu \mathrm{g} / \mathrm{ml}$} & \multicolumn{8}{|c|}{ Rifampicin concentration in $\mu \mathrm{g} / \mathrm{ml}$} \\
\hline & 0.025 & 0.05 & 0.1 & 0.20 & 0.5 & 1 & 2.5 & 5 & 1 & 2 & 4 & 8 & 16 & 32 & 64 & 128 \\
\hline $\begin{array}{l}\text { MIC of Plain } \\
\text { TB Drugs for } 60 \text { clinical } \\
\text { sample }\end{array}$ & 2 & 18 & 23 & 3 & 7 & 2 & 3 & 2 & $\mathbf{0}$ & $\mathbf{0}$ & 32 & $\mathbf{0}$ & 19 & 4 & 2 & 3 \\
\hline $\begin{array}{l}\text { MIC of Norbornene } \\
\text { based TB drug for } 60 \\
\text { clinical samples }\end{array}$ & 4 & 3 & 4 & 1 & 12 & 2 & 21 & 13 & 10 & 16 & 15 & 8 & 5 & 4 & 1 & 1 \\
\hline
\end{tabular}

It was observed that NOR-PEG-RIF showed anti-TB activity with lower concentration. We found that there is no significant antimycobacterial activity of NOR-PEG-INH $(\mathrm{P}=.14)$, but there is a significant antimycobacterial activity of NOR-PEG-RIF $(\mathrm{P}=.00)$ in comprison with plain Rifampicin.

Fig.1 Norbornene derived Isoniazid Copolymer

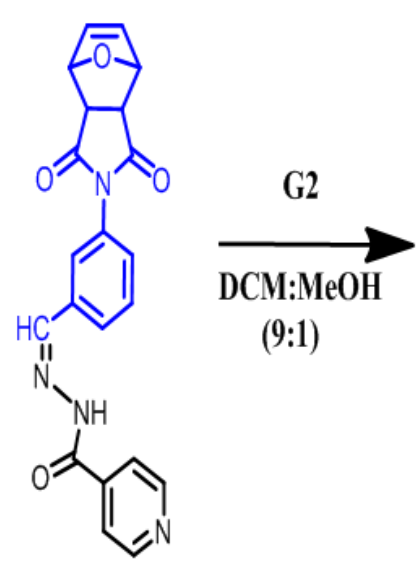

Norbornene anhydride + Isoniazid<smiles>C=CC(C)(C)C1OC(CC(C)(C)C)C2C(=O)N(c3cccc(/C=N\NC(=O)c4ccncc4)c3)C(=O)C21</smiles>

ROMP

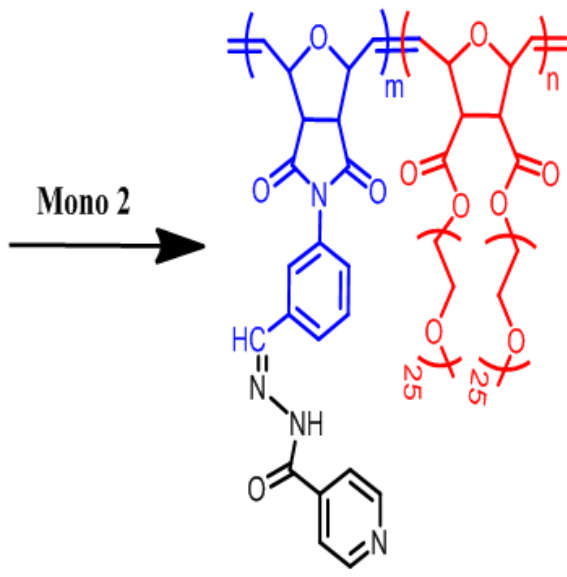

Norbornene+polyethyelene glycol+ Isoniazid

G2- Genetration 2, DCM- Dichloromethane, MeoH- Methanol, Mono2- Monomer 2, ROMP-Ring opening metatheses polymerization. 
Fig.2 Norbornene derived Rifampicin Copolymer

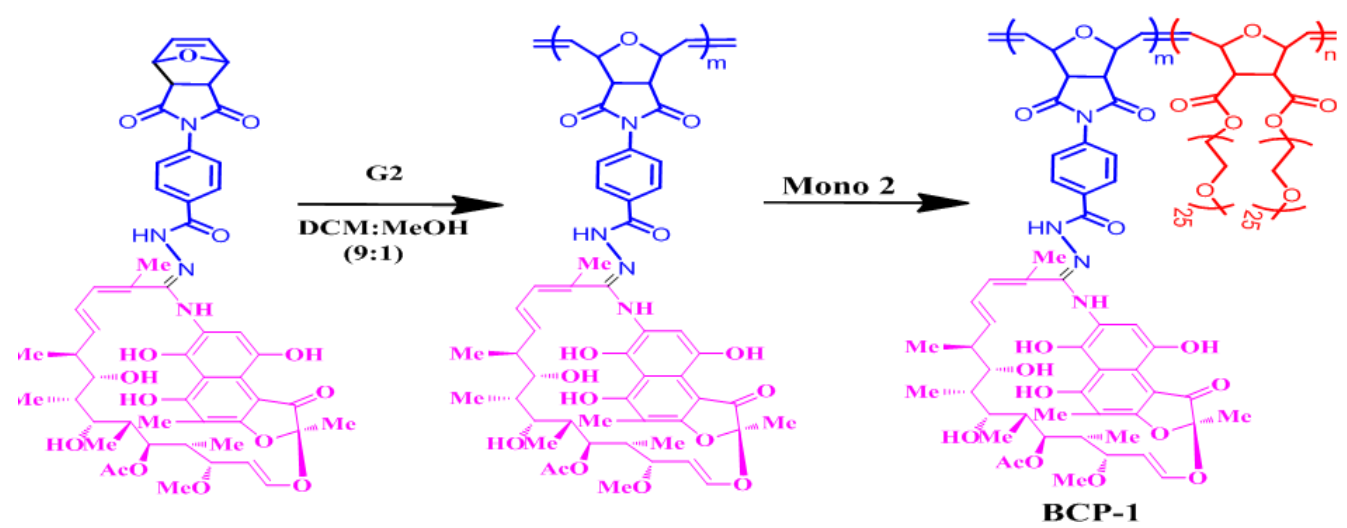

Norbornene anhydride + Isoniazid

ROMP

Norbornene+polyethyelene glycol+Rifampicin

G2- Genetration 2, DCM- Dichloromethane, MeoH- Methanol, Mono2- Monomer 2, BCP-1Block copolymer 1.

The MIC of Norbornene derived INH was around $1 \mu \mathrm{g} / \mathrm{ml}$ and for plain drug INH was around $0.025 \mu \mathrm{g} / \mathrm{ml}$, the MIC of Norbornene derived RIF was around $4 \mu \mathrm{g} / \mathrm{ml}$ and for plain RIF was around $16 \mu \mathrm{g} / \mathrm{ml}$. The nanodrug conjugates have high drug loading efficiency up to $58-95 \%$, so that more amounts of drugs which is proposed to act on target site can be loaded (Tatiany, 2012).

The invitro study it is proved that anti-TB drugs along with nanocarrier are stable under extracellular conditions (Vijayakameshwari, 2012). Norbornene derived isoniazid has showed lower MIC compared to plain drug Isoniazed, Some of the drugs losses their activity while binding to egg proteins, the ingredients of $\mathrm{LJ}$ medium ( $\mathrm{Yu}, 2011)$. Since Rifampicin shows lower MIC, Rifampicin resistance also called Multidrug resistance can be prevented by use of this nanocarrier which will additionally reduces the duration of treatment. Further studies are necessary to know the activity of Norbornene derived isoniazid and Rifampicin on lung tissue and cell line.
Cytotoxicity assay was done already (Shivasankar et al., 2014) and proved that at a lower concentrations of the drug, the cytotoxicity could not be attributed to damage to cytoplasmic, endosomal and lysosomal membranes, thus preventing normal human cell death.

In conclusion, the standardization of antimycobacterial susceptibility of nanocarrier with antituberculosis drugs revealed that Norbornene based isoniazid has lower MIC value thus it will gives better penetration into cell for inhibition. The MIC value was lower for Rifampicin with nanocarrier also for the inhibition. As the isoniazid and the Rifampicin are the main drugs utilized for the treatment of Tuberculosis infection and more concern on Multi drug Resistance Tuberculosis, if nanosized polymer with anti-tuberculosis drugs has an effective anti-mycobacterial activity then these drugs are of focus on future treatment protocol for Tuberculosis infection. 


\section{Acknowledgements}

The authors are grateful to acknowledge SRM University, Kancheepuram for the facilities to carry out the research, IISER Kolkata for providing the drug for the study and NIRT for providing strains and valuable suggestions towards the study.

\section{References}

Diacon, A.H., Pym, A., Grobuschi, M. 2014. Multidrug resistant Tuberculosis and culture conversion with Bedaquiline. N Eng. J .Med., 371: 723-732.

Heifets, L. 1988. Qualitative and quantitative drug-susceptibility tests in mycobacteriology. Am. Rev. Respir. Dis., 137: 1217-22. http://www.tballiance.org/downloads/ publications/TBA_Economics_Report .pdf. http://www.tbfacts.org/tbstatistics-india

Jing, J., Grant E.S., Vernon, M.S.O., Chuen, Li. 2008. Factors affecting therapeutic compliance: A review from the patient's perspective. Ther. Clin. Risk Manag., 4(1): 269-286.

Jong, W.H.D., Borm, P.J.A. 2008. Drug delivery and nanoparticles: Applications and hazards. Int. J. Nanomed., 3(2): 133-149.

Mehta, S.K., Neha, J. 2013. Formulation of Tyloxapol niosomes for encapsulation, stabilization and dissolution of anti-tubercular drugs Colloids and Surfaces B: Biointerfaces, 101: 434- 441.

Ming, Z., Claudia, S., Neeraj, D., Anthony, V., Vasan, K., and Sreevalli, S. 2014. .In Vitro and In Vivo Activities of Three Oxazolidinones against Nonreplicating Mycobacterium tuberculosis. Antimicrob. Agents Chemother., 58(6): 3217-3223.

NIRT Manual. 2010. Standard protocols for Mycobacteriology Laboratory,
Ormer, I.N. 2011. Development of new vaccines and drugs for TB: limitations and potential strategic errors. Future Microbiol., 6(2): 161-177.

Pratima, A.N. 2015. Nanotechnology and its Applications in Medicine. Med. chem., 5, 081-089.

Richard, J., O'Brien, M., and Spigelman, M., 2005. New Drugs for Tuberculosis: Current Status and Future Prospects. Clin Chest Med. 26, 327 -340.

Shivshankar, R.M., Himadri, D., Ashlin, S., Jaasri, D.S., Raja, S. 2014. Increased bioavailability of Rifampicin from stimuli responsive smart nano carrier. ACS, Appl. Mater. Interfaces, 6 (19), 16895-16902.

Shivshankar, R.M., Koushik, C., Himadri, D., Jayasri, D.S. and Raja, S. 2014. Stimuli responsive nanocarrier for an effective delivery of multifrontline tuberculosis drugs. Polym. Chem., 5: 2725-2735.

Shivshankar, R.M., Vijayakameswara, R.N., and Raja, S. 2011. Reversible $\mathrm{pH}$ and lipid sensitive vesicles from amphiphilic Norbornene-derived thiobarbiturate homopolymers. ACS Macrolett., 1: 482-488.

Skripconda, V., Danilovitis, M., and Pehme, L. 2013. Delamamnid Improves Outcomes and Reduces Mortality in Multidrug resistant Tuberculosis. Eur. Respir. J., 41: 1393-1400.

Svetlana, G., Kevin, K., Micheal, D., and Leonid, H. 2005. The potential advantages of nanoparticle drug delivery systems in chemotherapy. Is J Respir Crit Care Med., 172: 14871490.

Tatiany, J.D., Mariane, R., Nicole, M., Rodrigo, D.V., Vitor, J.A. and Lucia, A.G. 2012. An isoniazid analogue promotes Mycobacterium tuberculosis -nanoparticle interaction and enhances bacterial killing by 
macrophages. Antimic. Agents Chem., 2259-2267.

Vijayakameshwari, R., Shivshankar, R.M., Abhinoy, K., Jayasri, D.S. and Raja, S. 2012. Norbornene derived doxorubicin copolymers as drug carrires with $\mathrm{pH}$ responsive hydrazone linker. Biomacromol., 13: 221-230.

Yu, X., Jiang, G., Li, H., Zhao, Y., Zhang, H., and Zhao, L. 2011. Rifampin stability in $7 \mathrm{H} 9$ broth and Löwenstein-Jensen medium. J Clin Microbiol., 249(3): 784.

\section{How to cite this article:}

Kalaiselvi, K., V. Mangayarkarasi, N.S. Gomathi, R.M. Shivshankar and Raja Shunmugam. 2016. Antimycobacterial Activity of Norbornene-Polyethylene Glycol, Isoniazid and Rifampicin Nanocarrier towards Mycobacterium tuberculosis. Int.J.Curr.Microbiol.App.Sci. 5(8): 394-401. doi: http://dx.doi.org/10.20546/ijcmas.2016.508.042 